\title{
Key frame extraction using hybrid algorithm of dynamic sign language
}

\author{
Hussein Ali Aldelfy ${ }^{1}$, Mahmood Hamza Al-Mufraji ${ }^{2}$, Thamir R. Saeed ${ }^{3}$ \\ ${ }^{1,2,3}$ Department of Electrical Engineering, University of Technology, Iraq \\ ${ }^{1}$ Faculty of Engineering, University of Mustansiriyah, Baghdad, Iraq
}

\begin{tabular}{l} 
Article Info \\
\hline Article history: \\
Received Jun 10, 2018 \\
Revised Nov 19, 2018 \\
Accepted Nov 30, 2018 \\
\hline Keywords: \\
DWT \\
Edge detection \\
Hybrid \\
Key frame \\
Video
\end{tabular}

\begin{abstract}
Video summarization used in many applications such as video object recognition and classification. Different methods used for this purpose: key frame extraction using edge detection and key frame extraction using discrete wavelet transform (DWT). In video processing, numerous frames containing similar information, this leads to time consumption and slow processing speed and complexity. New method Hybrid DWT-Edge Key Frame Detection (HDEKFD) of dynamic sign language presented in this paper. This method combined two algorithms edge detection and DWT to increase processing speed by reduce the numbers of redundant frames. Simulation results illustrate the efficient of the proposed algorithm compare to other methods.
\end{abstract}

Copyright @ 2019 Institute of Advanced Engineering and Science. All rights reserved.

\section{Corresponding Author:}

Hussein Ali Aldelfy,

Department of Electrical Engineering,

University of Technology, Iraq.

Email: hussienaldulfy@gmail.com

\section{INTRODUCTION}

To extract valid information from video, without any loss of information, much attention is being paid to video processing technology. For this Key Frame is very useful technique. Extracting a small numbers of key frames that can abstract the content of video is very important for efficient browsing and retrieval in video database. Video processing techniques can be used to remove redundant frames from video so that it will solve the problem of the data storage [1]. Key frame extracting based on threshold technique where the calculated threshold is brought out in comparison with the difference histogram of the image [2]. An effective and efficient content based key frame detection method based on content analysis and feature detection from key frame proposed in [3]. Select and extract key frames from news video shot based on dissimilarity between each pair of consecutive frames was presented in [4]. Another approach for key frames extraction from video based on Faber-Schauder coefficients and singular value decomposition presented in [5] through the key frame sequence detection and identification, the sign language can be rapidly recognized. At the same time, sign language is a way for deaf people to communicate and exchange ideas through finger alphabet and gestures instead of language [6]. Key frame extraction method based on unsupervised clustering and mutual comparison were developed in [7]. A method of key frame extraction using thresholding of absolute difference of histogram of consecutive frames of video data was proposed [8]. Key frame extraction method using DWT wavelet statistics was proposed in [9]. The key frames are found by computing the edge difference between the consecutive frames and those frames exceeding the threshold are considered as key frame [10].

In this study, a New Method Hybrid DWT-Edge Key Frame Detection (HDEKFD) of dynamic sign language is proposed. This method combined two algorithms edge detection and DWT to increase processing speed by reduce the numbers of redundant frames. Simulation results illustrate the efficient of the proposed 
algorithm compare to other methods. This paper is organized as follows. Section 2 shows some method for key frame extraction. Section 3 presents the proposed method, and Section 4 briefly discusses the experimental results and Section 5 is the conclusion of this study in compare with the DWT wavelet method and the Edge detection method.

\section{GENERAL METHODS FOR KEY FRAME EXTRACTION}

This section described some of general methods used for key frame extraction:

\subsection{Key Frame Extraction Based on DWT Wavelet Statistics:}

The first step, the video frames are read and, then two consecutive frames transformed with DWT to obtain four sub bands, LL, HL, LH, and HH. Only three sub-bands, HL, LH, and HH are used to detect key frame. For each sub band different value is estimated by subtracting detail component values of current and next frame as shown in Equation 1.

$$
D_{l}(k)=\sum_{i=1}^{m} \sum_{j=1}^{n}\left(c H_{l+1}(i, j)-c H_{l}(i, j)\right) l=1,2,3
$$

where $\mathrm{i}$ and $\mathrm{j}$ are numbers of row and column, $\mathrm{cH}_{l}$ are the HL, $\mathrm{LH}$ and $\mathrm{HH}$ band of gray images $V_{k}$ and $\mathrm{cH}_{l+1}$ are the HL, LH and HH band of gray images $V_{k+1}$. In the second step, mean $\mu_{l}$ and standard deviation $\sigma_{l}$ are calculated from step one using difference values of sub bands Equation 2 and Equation 3.

$$
\begin{aligned}
\mu_{l} & =\frac{\sum_{i=1}^{N-1}\left(D_{l}(i)\right)}{N-1} \\
\sigma_{l} & =\operatorname{sqrt}\left[\frac{\sum_{i=1}^{N-1}\left(D_{l}(i)-\mu_{l}\right)^{2}}{N-1}\right]
\end{aligned}
$$

The third step, threshold value $\gamma_{l}$ for each sub band is computed by Equation 4 . the last step a comparison between threshold and difference values then if two difference values of any two sub bands are greater than each related threshold, the last frame considered as a key frame.

$$
\gamma_{l}=\mu_{l}+\beta . \sigma_{l}
$$

\subsection{Edge Detection}

In this method the video stream is taken as input convert into different images. MAfter this image is converted into edge detected using of one of the edge detection method. Canny detection method is used in this phase of key frame extraction. After that the edge difference between two successive frames are calculated and in this way the difference between all frames is calculated. Then the total accumulated difference is taken and by using that the mean of the total video is calculated. After that the standard deviation is calculated. The threshold is calculated depending on mean and standard deviation calculation then if the difference between two successive frames is greater than the threshold value the key frame is extracted. Figure 1 show the flowchart for key frame extraction using DWT method. Figure 2 shows the flow chart of the key frame extraction based on edge detection method.

\section{PROPOSED METHOD HYBRID DWT-EDGE KEY FRAME DETECTION}

The proposed method Hybrid DWT-Edge Key Frame Detection (HDEKFD) comprises two phase, the first phase is the key frame extraction based on discrete wavelet method. The output files from this phase is taken as input to the second phase that key frame extraction based on edge detection as shown in figure. Figure 3 show the the block diagram of key frame extraction based on (HDEKFD) method. 


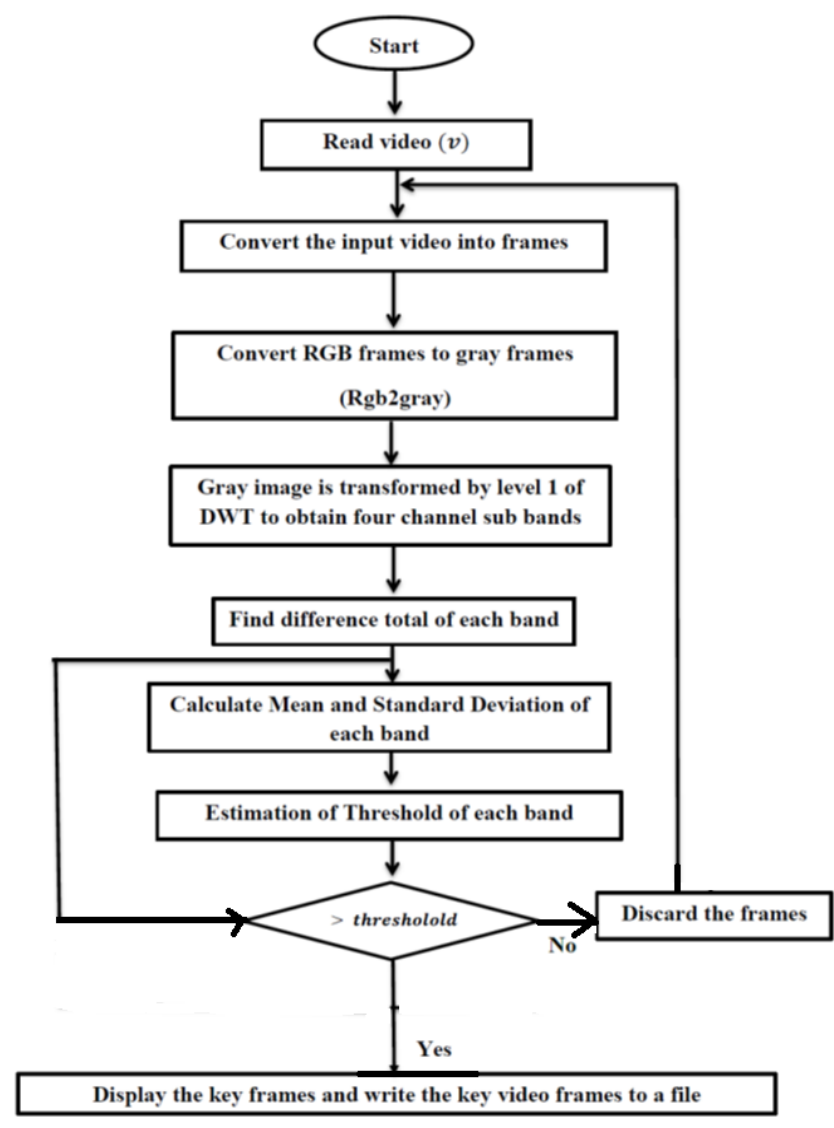

Figure 1. Flowchart for key frame extraction using DWT method. (Phase 1)

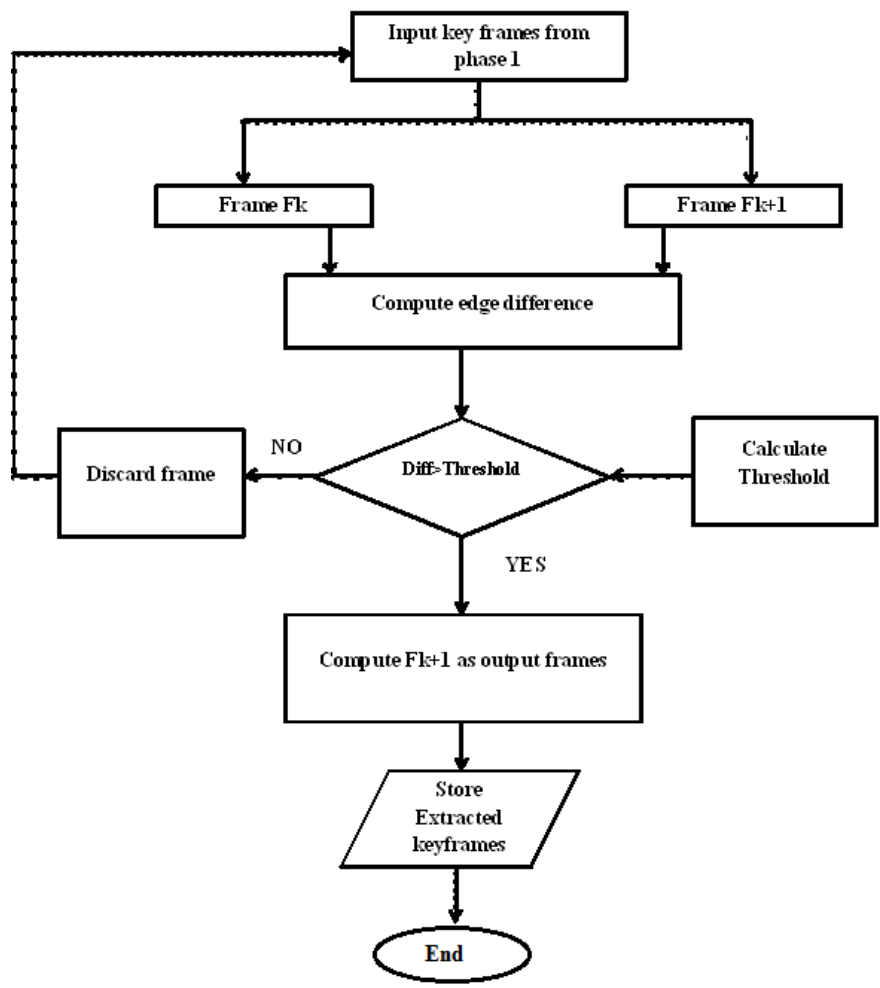

Figure 2. Flowchart for key frame extraction based on edge detection method (phase 2) 


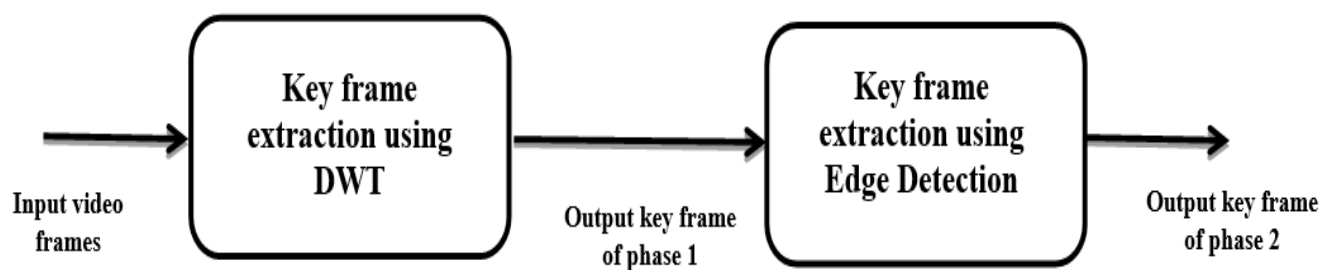

Figure 3. The block diagram of key frame extraction based on (HDEKFD) method

\section{THE EXPERIMENTAL RESULTS}

In this section of research is indicative words belong to Deaf and dumb people, these words were collected in Arabic language and Iraqi dialect and individual words in collaboration with Iraqi Ministry of labour and Social Affairs. More than forty words signalled task in dealing one word is used as an example, water word IS used as inputs to the proposed system also been using MATLAB program 2013b in the implementation of the proposed algorithm. The water video is $11.9 \mathrm{MHz}$ and 5 second time length and 30 frame /sec. The comparative results of key frame extraction based on (HDEKFD) method with other methods is illustrated In Table 1.

Table 1. Comparative Results

\begin{tabular}{ccc}
\hline Method & No of frames in video & Key frame No \\
\hline Discrete Wavelet method & 174 & 34 \\
Edge detection method & 174 & 27 \\
(HDEKFD) method & 174 & 10 \\
\hline
\end{tabular}

The key frame extraction of DWT wavelet method shown in Figure 4. The number of images about 34 image. Figure 5 shows the key frame extraction based on edge detection method and the number of images resulted about 27 images. Figure 6 shows the key frame extraction based on the (HDEKFD) proposed method and the number of images resulted about (10) images.

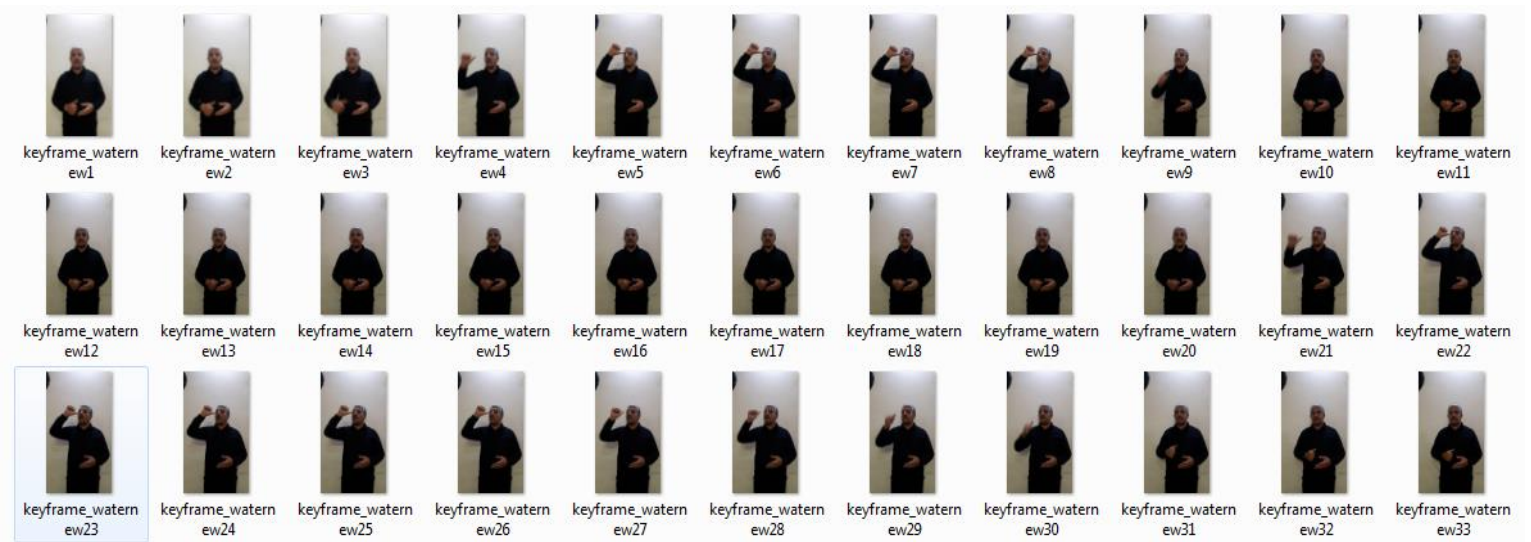

Figure 4. Key frame extraction based on discrete wavelet method 


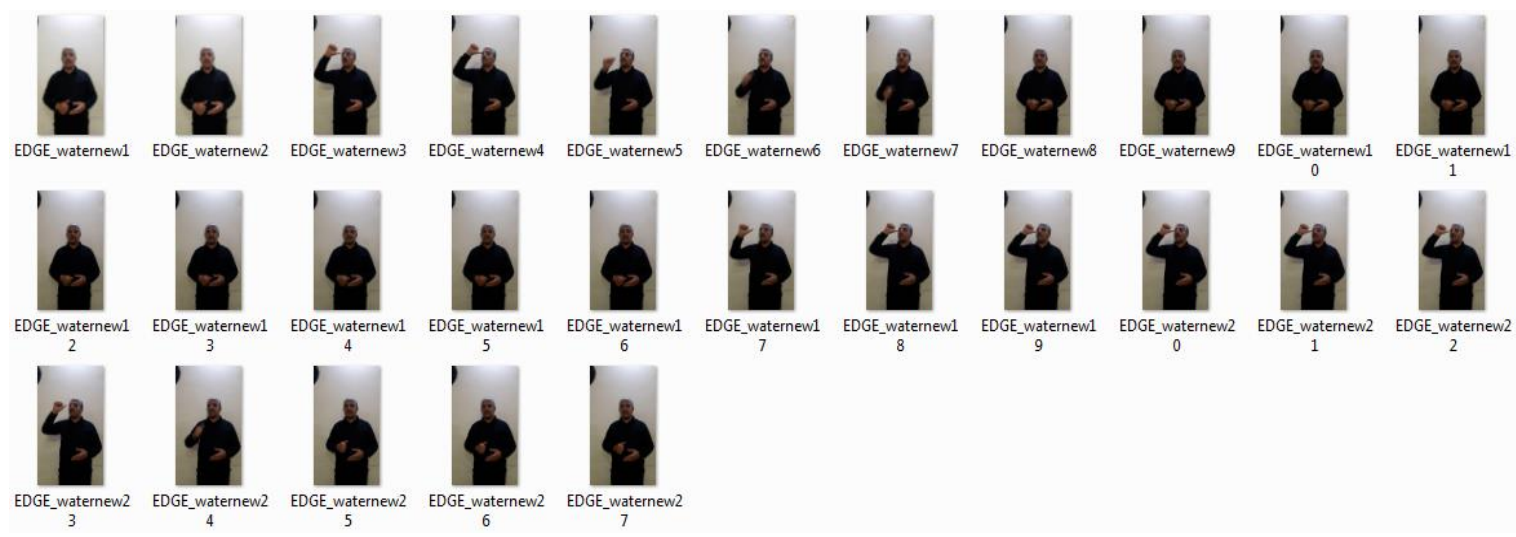

Figure 5. Key frame extraction based on Edge detection method

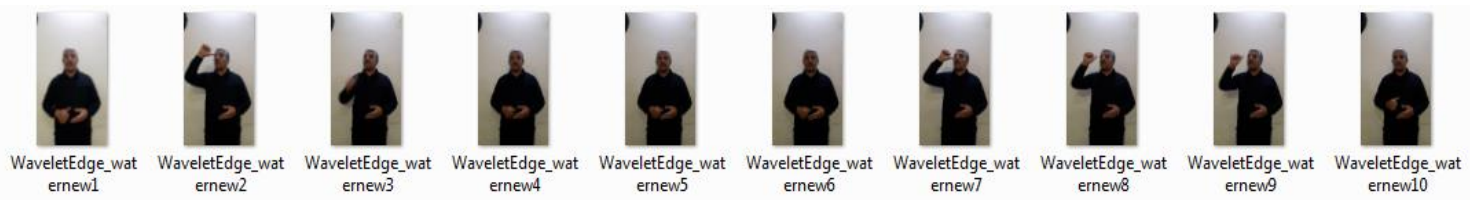

Figure 6. Key frame extraction based on HDEKFD method

\section{CONCLUSION}

In this paper key frame extraction based on (HDEKFD) method is presented. The Aim of this method is to reduce the redundant frames that can lead dimensionality reduction of feature vector classification of an isolated Arabic word for deaf and dump peoples. According to the experimental results, the (HDEKFD) Method is effective in reduction of key frames in comparison with two methods and this result leads to an effective reduction in time consuming in processing time and reduction of storage capacity. As our future work, we will continue in our research for pattern recognition of sign language based on the resulted key frames from a used video clips.

\section{REFERENCES}

[1] Amitkumar M. Satpute, K. R. Khadarkar" Video Summarization by Removing Duplicate Frames from Surveillance Video Using Keyframe Extraction" International Journal of Innovative Research in computer and Communication Engineering, Vol.5,pp.8501-8509, Issue 4,April 2017.

[2] Sanjoy Ghatak, "Key - Frame Extraction Using Thresholdtechnique", International journal of Engineering Applied Sciences and Technology, Vol. 1,Issue 8, ISSN No.2455-2143, pp.51-56, 2016.

[3] Zhenyu Wu, Ruiquing Wu,Hon gyang Yu, Bin Tang "Key Frame Extraction Towards Kernel -SIFT Identification" International Conference on Advanced Computer Science and Electronics Information, pp.163-166, (ICACSEI 2013).

[4] Sanjoy Ghatak, Debotosh Bhattacharjee "Extraction of Key Frames from News Video Using EDF, MDF AND HI Method for News Video Summarization “ International Journal of Engineering and Innovative Technology(IJEIT), Vol 2,pp.188-194, Issue 12,June 2013.

[5] Assma Azeroual, Karim Afdel, El Hajji Mohamed Hassan Douzi “ Key Frame Extraction using Faber -Schauder Wavelet" Advances in information science and computer Engineering. pp.317-322, 2015.

[6] L. Shurong, H. Yuanyuan, H. Zuojin, and D. Qun, "Key Frame Detection Algorithm based on Dynamic Sign Language Video for the Non Specific Population," International Journal of Signal Processing, Image Processing and Pattern Recognition, vol. 8, pp. 135-148, 2015.

[7] N. J. Janwe and K. K. Bhoyar, "Video Key-Frame Extraction using Unsupervised Clustering and Mutual Comparison," International Journal of Image Processing (IJIP), vol. 10, p. 73-84, 2016.

[8] C. Sheena and N. Narayanan, "Key-frame Extraction by Analysis of Histograms of Video Frames Using Statistical Methods," Procedia Computer Science, vol. 70, pp. 36-40, 2015.

[9] K. T. Tint and K. Soe, "Key frame extraction for video summarization using DWT wavelet statistics," International Journal of Advanced Research in Computer Engineering \& Technology (IJARCET), vol. 2, pp. pp: 1829-1833, 2013.

[10] K. Khurana and M. Chandak, "Key frame extraction methodology for video annotation," International Journal of Computer Engineering and Technology, vol. 4, pp. 221-228, 2013. 